\title{
Problems and Planning Analysis of Road and Street Reform in Old Town -- Taking Dalian Old Street as an Example
}

\author{
Xu Mingming \\ Dalian University of Technology City Institute, Dalian, 116600
}

Keywords: old town; road and street; transform; Dalian

\begin{abstract}
With the rapid development of the domestic economy, the development of urban construction is even more rapid. At the same time, there are a lot of problems in urban areas that need to be upgraded. Under the current construction situation, it is worth exploring in the renovation of road and street in old district. This paper analyzes and elaborates some problems existing in the reconstruction of road and street in old city, and puts forward some ideas, which will be helpful and enlightening to the project of road and street reform, including (1) rational division of the old street area function; (2) pedestrian street facade to form a unified style; (3) the combination of Chinese and Western, ancient and modern style, the mastery of artistic sketches and details; (4) the building and urban road between the reserve of about a few meters of outward swing commercial land; (5) three-dimensional parking lot to solve the problem of vehicle parking disorderly and other traffic problems.
\end{abstract}

\section{The significance of the transformation of the old city roads and streets}

From the aspects of improving residents' living environment, optimizing urban spatial layout, enhancing urban vitality and improving urban functions, the transformation of old neighborhoods is extremely necessary and inevitable. At the same time, road and street reconstruction is an uninterrupted process, which depends on the city's development goals and economic growth rate, is a long-term work.

The old city is the most concentrated area of cultural precipitation, and is full of the vitality of the original ecology. The distinctive and life-filled neighborhoods show the city's characteristics, history and diversity in the old city. Sharinin once said, "The city is an open book, from which you can see its ambitions." The renovation of the block is not simply to renovate the building facade, improve the living environment of the residents, improve the municipal facilities, but also shoulder the task of continuing the local culture and shaping the city's characteristics. It can be said that this kind of transformation can not only target individual blocks or buildings, nor can it only consider economic benefits. More needs to consider humanities, history, context and social comprehensive interests, and stand on the higher overall planning of the city. Look at the problem from a holistic perspective.

\section{Some problems and misunderstandings in the reconstruction of the old city roads and streets}

Some cities and regions blindly study big cities, blindly obsessed with the atmosphere of modern big cities and pursue economic benefits, while ignoring differences in traditional traditional architectural appearance, human history, climate characteristics, and urban nature. In the process of urban renewal, the pursuit of large-scale, speed-based construction, demolition more than transformation, and hastily to refine the façade of the block. The building adopts a large number of European and American styles. The direct consequence of replacing the local cultural practices with the foreign culture is that the traditional architectural style of the region gradually disappears into the flooded Western-style buildings, which also leads to the similarity of many neighborhoods and the cloning of thousands of streets. happens sometimes.

The warm house project in many cities in the northern region, the façade design not only ignores 
the original building façade, but also the large-scale greenhouse project often uses the same style of façade modeling, and the facades of the greenhouses in different districts are almost the same. Make the regional style extremely single and boring. These practices have greatly changed the characteristics of the old neighborhoods, neglected the embodiment and creation of the spirit of the neighborhood, and became a space of no connection, no sense of history and no sense of identity, eliminating people's original memories.

Many cities have self-growing commercial streets and night markets. It is bustling and lively. It is a city's landscape and a historical memory of the locals. In the renovation of the old streets, these are the top priorities in the transformation. Some cities even have their own night market shopping streets as tourist attractions, and the degree of attention can be seen.

However, it is often counterproductive. After many large-scale commercial streets have invested heavily in renovation, the flow of people has not risen and the business has been tepid. These remodeled commercial streets can be said to be completely new. The shop windows are clear and the roads are spotless. There are sculptures everywhere, rest benches, road signs, garbage boxes, exquisite and pleasant, plaques are neat and tidy, carefully designed green pieces embellish the surrounding, shopping environment Got a big boost. Fashion, taste, and style are all available, and you can take pictures on the street. What is the reason why the popularity is falling and consumers are not buying it? Summarizing the experience, simply saying: "the gas of the city" disappears, the ground is not grounded, and the positioning of the commercial street is blurred.

When it comes to the old streets, many people will think of elements such as "original ecology", "low price, strange", "outside small street vendors" and "street food stalls". These are the fundamental reasons for the prosperity of most of the old night markets, and are an important factor influencing the flow of a large number of people around. From a commercial orientation, the commercial complex and high-end shopping malls of the one-stop shopping experience have their own consumer groups. For the old street after the renovation, the atmosphere of the original traditional block disappeared. Will the original old street residents be interested in the existing old street? The decline of local people's flow will definitely reduce the interest of foreign tourists to come and play. The reduction of tourists outside the region will further stimulate the reduction of local people's flow, and the cycle will be repeated repeatedly, resulting in a sharp drop in people flow.

For shoppers, going to the old street is a lively atmosphere, eating snacks, looking around the life-filled alleys, looking for some interesting gadgets. The decline of the "city well" feature has led to a lack of consumer interest, which is the main factor in the decline in the popularity of Old Street. In addition, the commercial management fees and the increase in the general rent after the renovation of the block have also directly increased the operating costs of the merchants and led to the rise in commodity prices, which is one of the reasons for the decrease in traffic.

For the self-growing commercial and residential mixed-style business of Laojie, it is indispensable for the stalls outside the shops to be widely distributed. This kind of business atmosphere is not only close to the people but also popular.

However, due to the appearance of the city and the consideration of transportation, it is often a blow to the attitude of urban management. At the same time, the regulation of the new block also tends to neglect the land occupation needs of the stalls, so that even the new block buildings will still face the intermittent business of the merchants. The swaying business and urban management become two opposites.

In this regard, the author believes that the external swing booth has greatly promoted the commercial atmosphere, and at the same time, the simple ban can not solve the problem fundamentally, and the merchants who conduct guerrilla warfare with the urban management are proof. Treating the outside stalls should be guided under the general principle of urban design to give space for business. For example, the design of the parasols, tables and chairs, surrounding plaques and green plants landscapes makes the pendulum booth a city landscape.

At the same time, it is recommended to reserve a few meters of sway commercial land between buildings and urban roads at the level of control, which can greatly alleviate the contradiction 
between commercial business needs and urban image.

Due to historical reasons, the spatial form of Lao Cai is affected by the daily activities of the surrounding residents. It often includes residential buildings, shops, hospitals and schools in a small area of walking scale. These naturally formed small-scale neighborhoods are not only feature-rich, but also close the distance between residents in the neighborhood. At the same time, small-scale streets cause short distances and a large number of intersection corners, which also enriches the spatial shape of the blocks, making the old streets Being energetic and energetic has become a major feature of the city.

However, the current old street renovation is often influenced by modern planning concepts. The old block is converted into a new block. The road plan is based on motorized traffic, which makes the road scale in the planning of the new block far larger than the traditional block. The increase in block size leads to a decrease in the accessibility of the block, which affects the permeability of space and space, reduces the interest of the block space and the harmonious order of the old street.

In addition, in the current urban master plan, the simple land use classification can not adapt to the multi-functional composite area of the old city old street. The simple division of different land use attributes has resulted in the separation of people's living space, the simplification of living space, and the disappearance of neighborhoods. These factors have caused the annihilation of the characteristics of the block, the decline of vitality, and the small-scale multi-functional composite living environment full of local characteristics no longer exists.

Builders should dialectically look at the conflict between modern planning concepts and the transformation of old urban and old streets. This spontaneous mixed multi-functional attribute of Laojie often brings a series of related problems such as chaotic layout, poor environment, lack of development space, low land use efficiency and infrastructure lag. These factors hinder the development of the city, and can not adapt to a series of problems such as the rapid increase of urban population and traffic congestion. However, if it is simply influenced by the construction mode of the big city and ignores its own characteristics and historical context, it will have a bad influence on urban characteristics and historical memory. Therefore, the planning and renovation of the old streets of the old city should be dynamic, technical, social and long-term.

\section{The transformation process should ensure the continuity of the personality of the road street}

The personality of the block is simply a feature. It blends in all aspects of the neighborhood, architecture, landscape, lifestyle of the residents, and more. No matter which aspect, we can deeply feel and appreciate the unique feelings of a region. The continuation of personality is a continuation of history and culture, and it is a message and memory that conveys history to us. In particular, some historical districts with a long history and complete protection can fully describe the life, work, entertainment and other aspects of that era. The formation of this personality, influenced by nature and human activities, is contained in a variety of carriers. This is the carrier of history, a memory that cannot be copied and precious.

Objectively speaking, the road and street reconstruction process will inevitably encounter problems of demolition and reconstruction, transformation and protection, and the relationship between the past and the present. The development of the city and the protection of the old streets are dialectical and unified. People's nostalgia for urban history, dependence on traditional culture and old-fashioned psychology require us to protect the historical buildings of urban blocks, and have appropriate respect for the traditional architectural forms of the original areas to achieve the continuity of personality. The personality of the block also changes with the development of the times. The transformation should try to combine the old and the new, and promote the diversified development of the whole region. 


\section{Diversity and sustainable development of roads and streets in the old city}

Block renovation and protection should be diverse. It should not be single. It should not use formulas and rules to fix the development of neighborhoods. Instead, it should try to understand and absorb the unique culture and history of the region and create a diverse, harmonious and full. An area of life. We should understand that the history, characteristics, and natural landscape of a region have been formed by centuries or even thousands of years. Builders should not be arrogant to think that modernity is correct, that is, it must transcend the wisdom of predecessors.

The benign self-renewal and sustainable development of the block is not simply to eliminate the decay space, but to find the cause before the decline, maintain the vitality of the city, and improve the quality of life of the residents. Sustainability emphasizes the transformation of function and environment based on the existing functions of the neighborhood. It is different from traditional spatial expansion and large-scale reconstruction, but based on the changes in social and economic development and the life of ordinary people in society. In short, integrating material resources (living materials, building materials) and non-material (cultural, historical) resources, protecting non-renewable resources (natural landscapes, historical buildings) is the basic principle and the most effective way to maintain sustainable development.

As a basic element of the city, the street and street renewal is only one point in the urban change. These changes are not closed, nor are they static. They are the composition of modern life. Therefore, whether it is the inheritance of urban culture or the replacement of modern architecture, it should be based on the premise of "making the city better" and based on the human feeling. Therefore, the principles of road and street reconstruction are: first, to protect and inherit urban culture; second, to fully meet the needs of modern urban life. Purification is one aspect, and evolution is another. Only in this way can the city's self-renewal be sustainable and healthy.

5 Dalian is a tourist city with unique cultural characteristics. The fashion art is both creative and has many urban symbols. The new district and the historical old street coexist. There are many traditional neighborhoods, and there are also their own characteristics and problems.

Taking Laojie Street District of Dalian Sha District as an example, the existing commercial business is relatively complete, but it lacks unified management and has no unified image. It basically forms a spontaneous formation of the people and can gather a certain popularity, but wants to become a city-specific neighborhood and attract Certain tourists also need to manage and renovate in many problems: 1) lack of cultural atmosphere, no support of characteristic ideas 2) inconsistent design, lack of uniform packaging design and color management 3) lack of theme function classification, commercial form The theme store is single 4) The special food and the sale of featured products are scattered, and there is no uniform specification.

In the process of transformation, in order to ensure the continuity of the personality of the old street and the sustainable development of diversity, we need to grasp the principles in the following aspects:

1) Reasonable regional function division of Laojie, according to the surrounding environment and human flow analysis, it is divided into cultural industry product block, folk custom street, special food street, marine cultural product exhibition area and industrial exhibition display area.

2) The façade of the pedestrian street forms a unified style, and the planning and management are unified on the basis of retaining the original structure of the original building. Different main styles and colors are created for different neighborhoods, and special planning and management are carried out.

3) Combining Chinese and Western styles, combining ancient and modern, paying attention to the grasp of artistic sketches and details, and increasing the traditional culture and folk art of the neighborhood.

4) Reserve a few meters of sloping commercial land between the building and the urban road land. Consumers will sit on the street and the store will be open-air to create a four-season commercial pedestrian street.

5) In order to solve the traffic problems such as chaos and chaos, set up a special 
three-dimensional parking lot in a specific suitable area, and implement the diversion of people and vehicles to create a comfortable and safe shopping environment.

\section{References}

[1] Zhou Wenjun. Analysis and planning of problems in old city renewal. Construction engineering technology and design. 25th issue, 2014.

[2] Liang Min. The current situation of urban walking blocks and the planning and design countermeasures. China new technology and new products.2011, 11th issue.

[3] Liu Sisi. From the old city reconstruction and new city development to explore the relationship between China's urban development planning concept. Residential technology. 2009 (12).

[4] He Lihua. Design and research of street building facade from the perspective of urban design. Wuhan university of technology.2014.

Xu Ming-ming, (1986.12--), female, Han nationality, Dalian people in Liaoning, master, lecturer, Research direction: Landscape Architecture Planning and design 